\title{
Contraction After Small Transients *
}

\author{
Michael Margaliot ${ }^{\mathrm{a}}$ Eduardo D. Sontag ${ }^{\mathrm{b}}$ Tamir Tuller ${ }^{\mathrm{c}}$ \\ ${ }^{a}$ School of Elec. Eng.-Systems, Tel Aviv University, Israel 69978. Email: michaelm@eng.tau.ac.il \\ ${ }^{\mathrm{b}}$ Dept. of Mathematics and the Center for Quantitative Biology, Rutgers University, Piscataway, NJ 08854, USA. Email: \\ eduardo.sontag@rutgers.edu \\ ${ }^{\mathrm{c}}$ Dept. of Biomedical Eng., Tel Aviv University, Israel 69978. Email: tamirtul@yahoo.com
}

\begin{abstract}
Contraction theory is a powerful tool for proving asymptotic properties of nonlinear dynamical systems including convergence to an attractor and entrainment to a periodic excitation. We consider three generalizations of contraction with respect to a norm that allow contraction to take place after small transients in time and/or amplitude. These generalized contractive systems (GCSs) are useful for several reasons. First, we show that there exist simple and checkable conditions guaranteeing that a system is a GCS, and demonstrate their usefulness using several models from systems biology. Second, allowing small transients does not destroy the important asymptotic properties of contractive systems like convergence to a unique equilibrium point, if it exists, and entrainment to a periodic excitation. Third, in some cases as we change the parameters in a contractive system it becomes a GCS just before it looses contractivity with respect to a norm. In this respect, generalized contractivity is the analogue of marginal stability in Lyapunov stability theory.
\end{abstract}

Key words: Differential analysis, contraction, stability, entrainment, phase locking, systems biology.

\section{Introduction}

Differential analysis is based on studying the time evolution of the distance between trajectories emanating from different initial conditions. A dynamical system is called contractive if any two trajectories converge to one other at an exponential rate. This implies many desirable properties including convergence to a unique attractor (if it exists), and entrainment to periodic excitations (Lohmiller and Slotine (1998); Russo et al. (2010); Aminzare and Sontag (2014)). Contraction theory proved to be a powerful tool for analyzing nonlinear dynamical systems, with applications in

control theory (Lohmiller and Slotine (2000)), observer design (Bonnabel et al. (2011)), synchronization of coupled oscillators (Wang and Slotine (2005)), and more. Recent extensions include: the notion of partial contraction (Slotine (2003)), analyzing networks of interacting

\footnotetext{
* An abridged version of this note was presented at the 2014 IEEE CDC (Sontag et al. (2014)). The research of MM and TT is partly supported by a research grant from the Israeli Ministry of Science, Technology and Space. EDS's work is supported in part by grants NIH 1R01GM100473, AFOSR FA9550-14-1-0060, and ONR N00014-13-1-0074.
}

agents using contraction theory (Russo et al. (2013); Arcak (2011)), a Lyapunov-like characterization of incremental stability (Angeli (2002)), and a LaSalle-type principle for contractive systems (Forni and Sepulchre (2014)). There is also a growing interest in design techniques providing controllers that render control systems contractive or incrementally stable; see, e.g. (Zamani et al. (2013)) and the references therein, and also the incremental ISS condition in (Desoer and Haneda (1972)).

A contractive system with added diffusion terms or random noise still satisfies certain asymptotic properties (Aminzare and Sontag (2013); Pham et al. (2009)). In this respect, contraction is a robust property.

In this note, we introduce three forms of generalized contractive systems (GCSs). These are motivated by requiring contraction with respect to a norm to take place only after arbitrarily small transients in time and/or amplitude. Our work was motivated by certain models from systems biology that are not contractive with respect to any (fixed) norm, yet are "almost" contractive. One example is where contraction is lost only on the boundary of the state space, but trajectories emanating from this boundary "immediately" enter the interior of the state space. Thus, we have contraction after an arbitrar- 
ily short time transient. The goal of the note is to rigorously define these forms of contraction, study its properties, and derive sufficient conditions for its existence. The contribution of this note is thus two-fold: the theoretical study of this type of contraction after an infinitesimal transient, and using this notion to prove important asymptotic properties in applications. Indeed, contraction is usually used to prove asymptotic properties, and thus allowing (arbitrarily small) transients seems reasonable. We provide several sufficient conditions for a system to be a GCS. These conditions are checkable, and we demonstrate their usefulness using several examples of systems that are not contractive with respect to any norm, yet are GCSs.

In some cases, as we change the parameters in a contractive system it becomes a GCS just before it looses contractivity. In this respect, a GCS is the analogue of marginal stability in Lyapunov stability theory.

We begin with a brief review of some ideas from contraction theory. See (Soderlind (2006); Jouffroy (2005); Rüffer et al. (2013)) for more details, including the historic development of contraction theory, and the relation to other notions.

Consider the time-varying system

$$
\dot{x}=f(t, x),
$$

with the state $x$ evolving on a positively invariant convex set $\Omega \subseteq \mathbb{R}^{n}$. We assume that $f(t, x)$ is differentiable with respect to $x$, and that both $f(t, x)$ and $J(t, x):=\frac{\partial f}{\partial x}(t, x)$ are continuous in $(t, x)$. Let $x\left(t, t_{0}, x_{0}\right)$ denote the solution of (1) at time $t \geq t_{0}$ with $x\left(t_{0}\right)=x_{0}$ (for the sake of simplicity, we assume from here on that $x\left(t, t_{0}, x_{0}\right)$ exists and is unique for all $t \geq t_{0} \geq 0$ and all $x_{0} \in \Omega$ ).

We say that (1) is contractive on $\Omega$ with respect to a norm $|\cdot|: \mathbb{R}^{n} \rightarrow \mathbb{R}_{+}$if there exists $c>0$ such that

$$
\left|x\left(t_{2}, t_{1}, a\right)-x\left(t_{2}, t_{1}, b\right)\right| \leq \exp \left(-\left(t_{2}-t_{1}\right) c\right)|a-b|
$$

for all $t_{2} \geq t_{1} \geq 0$ and all $a, b \in \Omega$. In other words, any two trajectories contract to one another at an exponential rate. This implies in particular that the initial condition is "quickly forgotten". Note that Lohmiller and Slotine (1998) provide a more general and intrinsic definition, where contraction is with respect to a timeand state-dependent metric $M(t, x)$. Simpson-Porco and Bullo (2014) provide a general treatment of contraction on a Riemannian manifold; see also (Lewis (1949)). Some of the results below may be stated using this more general framework. But, for a given dynamical system finding such a metric may be difficult; see e.g. Aylward et al. (2008) for an algorithm for finding such contraction metrics using sum-of-squares programming.
Another extension of contraction is incremental stability (Angeli (2002)). Our approach is based on the fact that there exists a simple sufficient condition guaranteeing (2), so generalizing (2) appropriately leads to checkable sufficient conditions for a system to be a GCS. Another advantage of our approach is that a GCS retains the important property of entrainment to periodic signals.

Recall that a vector norm $|\cdot|: \mathbb{R}^{n} \rightarrow \mathbb{R}_{+}$induces a matrix measure $\mu: \mathbb{R}^{n \times n} \rightarrow \mathbb{R}$ defined by $\mu(A):=$ $\lim _{\epsilon \downarrow 0} \frac{1}{\epsilon}(\|I+\epsilon A\|-1)$, where $\|\cdot\|: \mathbb{R}^{n \times n} \rightarrow \mathbb{R}_{+}$is the matrix norm induced by $|\cdot|$. A standard approach for proving (2) is based on bounding some matrix measure of the Jacobian $J$. Indeed, it is well-known (Russo et al. (2010)) that if there exist a vector norm $|\cdot|$ and $c>0$ such that the induced matrix measure $\mu: \mathbb{R}^{n \times n} \rightarrow \mathbb{R}$ satisfies $\mu(J(t, x)) \leq-c$, for all $t_{2} \geq t_{1} \geq 0$ and all $x \in \Omega$ then (2) holds. (This is in fact a particular case of using a Lyapunov-Finsler function to prove contraction (Forni and Sepulchre (2014)).)

It is well-known (Vidyasagar, 1978, Ch. 3) that the matrix measure induced by the $L_{1}$ vector norm is

$$
\mu_{1}(A)=\max \left\{c_{1}(A), \ldots, c_{n}(A)\right\}
$$

where

$$
c_{j}(A):=A_{j j}+\sum_{\substack{1 \leq i \leq n \\ i \neq j}}\left|A_{i j}\right|,
$$

i.e., the sum of the entries in column $j$ of $A$, with non diagonal elements replaced by their absolute values. The matrix measure induced by the $L_{\infty}$ norm is $\mu_{\infty}(A)=$ $\max \left\{d_{1}(A), \ldots, d_{n}(A)\right\}$, where

$$
d_{j}(A):=A_{j j}+\sum_{\substack{1 \leq i \leq n \\ i \neq j}}\left|A_{j i}\right|,
$$

i.e., the sum of the entries in row $j$ of $A$, with non diagonal elements replaced by their absolute values.

Often it is useful to work with scaled norms. Let $|\cdot|_{*}$ be some vector norm, and let $\mu_{*}: \mathbb{R}^{n \times n} \rightarrow \mathbb{R}$ denote its induced matrix measure. If $P \in \mathbb{R}^{n \times n}$ is an invertible matrix, and $|\cdot|_{*, P}: \mathbb{R}^{n} \rightarrow \mathbb{R}_{+}$is the vector norm defined by $|z|_{*, P}:=|P z|_{*}$ then the induced matrix measure is $\mu_{*, P}(A)=\mu_{*}\left(P A P^{-1}\right)$.

One important implication of contraction is entrainment to a periodic excitation. Recall that $f: \mathbb{R}_{+} \times \Omega \rightarrow \mathbb{R}^{n}$ is called $T$-periodic if $f(t, x)=f(t+T, x)$ for all $t \geq 0$ and all $x \in \Omega$. Note that for the system $\dot{x}(t)=f(u(t), x(t))$, with $u$ an input (or excitation) function, $f$ will be $T$ periodic if $u$ is a $T$-periodic function. It is well-known (Lohmiller and Slotine (1998); Russo et al. (2010)) that if (1) is contractive and $f$ is $T$-periodic then for any $t_{1} \geq$ 
0 there exists a unique periodic solution $\alpha:\left[t_{1}, \infty\right) \rightarrow \Omega$ of (1), of period $T$, and every trajectory converges to $\alpha$. Entrainment is important in various applications ranging from biological systems (Margaliot et al. (2014); Russo et al. (2010)) to the stability of a power grid (Dorfler and Bullo (2012)). Note that for the particular case where $f$ is time-invariant, this implies that if $\Omega$ contains an equilibrium point $e$ then it is unique and all trajectories converge to $e$.

The remainder of this note is organized as follows. Section 2 presents three generalizations of (2). Section 3 details sufficient conditions for their existence, and describes their implications. Due to space limitations, the proofs of all the results are placed at: http://arxiv . org/abs/1506.06613

\section{Definitions of contraction after small tran- sients}

We begin by defining three generalizations of (2).

Definition 1 The time-varying system (1) is said to be:

- contractive after a small overshoot and short transient (SOST) on $\Omega$ w.r.t. a norm $|\cdot|: \mathbb{R}^{n} \rightarrow \mathbb{R}_{+}$if for each $\varepsilon>0$ and each $\tau>0$ there exists $\ell=\ell(\tau, \varepsilon)>0$ such that

$$
\begin{aligned}
& \left|x\left(t_{2}+\tau, t_{1}, a\right)-x\left(t_{2}+\tau, t_{1}, b\right)\right| \\
& \leq(1+\varepsilon) \exp \left(-\left(t_{2}-t_{1}\right) \ell\right)|a-b|
\end{aligned}
$$

for all $t_{2} \geq t_{1} \geq 0$ and all $a, b \in \Omega$.

- contractive after a small overshoot $(S O)$ on $\Omega$ w.r.t. a norm $|\cdot|: \mathbb{R}^{n} \rightarrow \mathbb{R}_{+}$if for each $\varepsilon>0$ there exists $\ell=$ $\ell(\varepsilon)>0$ such that

$$
\begin{aligned}
& \left|x\left(t_{2}, t_{1}, a\right)-x\left(t_{2}, t_{1}, b\right)\right| \\
& \quad \leq(1+\varepsilon) \exp \left(-\left(t_{2}-t_{1}\right) \ell\right)|a-b|
\end{aligned}
$$

for all $t_{2} \geq t_{1} \geq 0$ and all $a, b \in \Omega$.

- contractive after a short transient $(S T)$ on $\Omega$ w.r.t. a norm $|\cdot|: \mathbb{R}^{n} \rightarrow \mathbb{R}_{+}$if for each $\tau>0$ there exists $\ell=$ $\ell(\tau)>0$ such that

$$
\begin{aligned}
& \left|x\left(t_{2}+\tau, t_{1}, a\right)-x\left(t_{2}+\tau, t_{1}, b\right)\right| \\
& \leq \exp \left(-\left(t_{2}-t_{1}\right) \ell\right)|a-b|
\end{aligned}
$$

for all $t_{2} \geq t_{1} \geq 0$ and all $a, b \in \Omega$.

The definition of SOST is motivated by requiring contraction at an exponential rate, but only after an (arbitrarily small) time $\tau$, and with an (arbitrarily small) overshoot $(1+\varepsilon)$. However, as we will see below when the convergence rate $\ell$ may depend on $\varepsilon$ a somewhat richer behavior may occur. The definition of SO is similar to that of SOST, yet now the convergence rate $\ell$ depends only on $\varepsilon$, and there is no time transient $\tau$ (i.e., $\tau=0$ ). In other words, SO is a uniform (in $\tau$ ) version of SOST. The third definition, ST, allows the contraction to "kick in" only after a time transient of length $\tau$.

It is clear that every contractive system is SOST, SO, and ST. Thus, all these notions are generalizations of contraction. Also, both SO and ST imply SOST and, as we will see below, under a mild technical condition on (1) SO and SOST are equivalent. Fig. 1 on p. 8 summarizes the relations between these GCSs (as well as other notions defined below).

One motivation for these definitions stems from the fact that important applications of contraction are in proving asymptotic properties. For example, proving that an equilibrium point is globally attracting or that the statevariables entrain to a periodic excitation. These properties describe what happens as $t \rightarrow \infty$, and so it seems natural to generalize contraction in a way that allows initial transients in time and/or amplitude.

The next simple example demonstrates a system that does not satisfy (2), but is a GCS.

Example 1 Consider the scalar time-varying system $\dot{x}(t)=-\alpha(t) x(t)$, with the state $x$ evolving on $\Omega:=[-1,1]$, and $\alpha: \mathbb{R}_{+} \rightarrow \mathbb{R}_{+}$is a class $K$ function (i.e. $\alpha$ is continuous and strictly increasing, with $\alpha(0)=0)$. It is straightforward to show that this system does not satisfy (2) w.r.t. any norm (note that the Jacobian $J(t)=-\alpha(t)$ satisfies $J(0)=0)$, yet it is $S T$, with $\ell(\tau)=\alpha(\tau)>0$, for any given $\tau>0$.

\section{$3 \quad$ Main Results}

The next three subsections study the three forms of GCSs defined above.

\section{Contraction after a small overshoot and short} transient

Just like contraction, SOST implies entrainment to a periodic excitation.

Proposition 1 Suppose that the time-varying system (1), with state $x$ evolving on a compact and convex state-space $\Omega \subset \mathbb{R}^{n}$, is SOST, and that the vector field $f$ is T-periodic. Then for any $t_{0} \geq 0$ it admits a unique periodic solution $\gamma:\left[t_{0}, \infty\right) \rightarrow \Omega$ with period $T$, and $x\left(t, t_{0}, a\right)$ converges to $\gamma$ for any $a \in \Omega$.

Since both SO and ST imply SOST, Proposition 1 holds for all three forms of GCSs.

Our next goal is to derive a sufficient condition for SOST. One may naturally expect that if (1) is contractive w.r.t. 
a set of norms $|\cdot|_{\zeta}$, with, say $\zeta \in(0, p], p>0$, and that $\lim _{\zeta \rightarrow 0}|\cdot|_{\zeta}=|\cdot|$ then (1) is a GCS w.r.t. the norm $|\cdot|$. In fact, this can be further generalized by requiring (1) to be contractive w.r.t. $|\cdot|_{\zeta}$ only on suitable subset $\Omega_{\zeta}$ of the state-space. This leads to the following definition.

Definition 2 System (1) is said to be nested contractive $(N C)$ on $\Omega$ with respect to a norm $|\cdot|$ if there exist convex sets $\Omega_{\zeta} \subseteq \Omega$, and norms $|\cdot|_{\zeta}: \mathbb{R}^{n} \rightarrow \mathbb{R}_{+}$, where $\zeta \in(0,1 / 2]$, such that the following conditions hold.

(a) $\cup_{\zeta \in(0,1 / 2]} \Omega_{\zeta}=\Omega$, and $\Omega_{\zeta_{1}} \subseteq \Omega_{\zeta_{2}}$, for all $\zeta_{1} \geq \zeta_{2}$.

(b) For every $\tau>0$ there exists $\zeta=\zeta(\tau) \in(0,1 / 2]$, with $\zeta(\tau) \rightarrow 0$ as $\tau \rightarrow 0$, such that for every $a \in \Omega$ and every $t_{1} \geq 0$

$$
x\left(t, t_{1}, a\right) \in \Omega_{\zeta}, \quad \text { for all } t \geq t_{1}+\tau,
$$

and (1) is contractive on $\Omega_{\zeta}$ with respect to $|\cdot|_{\zeta}$.

(c) The norms $|\cdot|_{\zeta}$ converge to $|\cdot|$ as $\zeta \rightarrow 0$, i.e., for every $\zeta>0$ there exists $s=s(\zeta)>0$, with $s(\zeta) \rightarrow 0$ as $\zeta \rightarrow 0$, such that $(1-s)|y| \leq|y|_{\zeta} \leq(1+s)|y|$, for all $y \in \Omega$.

Eq. (7) means that after an arbitrarily short time every trajectory enters and remains in a subset $\Omega_{\zeta}$ of the state space on which we have contraction with respect to $|\cdot|_{\zeta}$. We can now state the main result in this subsection.

Theorem 1 If the system (1) is NC w.r.t. the norm $|\cdot|$ then it is SOST w.r.t. the norm $|\cdot|$.

The next example demonstrates Theorem 1. It also shows that as we change the parameters in a contractive system, it may become a GCS when it hits the "verge" of contraction (as defined in (2)). This is reminiscent of an asymptotically stable system that becomes marginally stable as it looses stability.

Example 2 Consider the system

$$
\begin{aligned}
\dot{x}_{1} & =g\left(x_{n}\right)-\alpha_{1} x_{1}, \\
\dot{x}_{2} & =x_{1}-\alpha_{2} x_{2}, \\
\dot{x}_{3} & =x_{2}-\alpha_{3} x_{3}, \\
& \vdots \\
\dot{x}_{n} & =x_{n-1}-\alpha_{n} x_{n},
\end{aligned}
$$

where $\alpha_{i}>0$, and $g(u):=\frac{1+u}{k+u}$, with $k>1$. This models a simple biochemical feedback control circuit for protein synthesis in the cell (Smith, 1995, Ch. 4). The $x_{i}$ s represent concentrations of various macro-molecules in the cell and therefore must be non-negative. It is straightforward to verify that $x(0) \in \mathbb{R}_{+}^{n}$ implies that $x(t) \in \mathbb{R}_{+}^{n}$ for all $t \geq 0$.

$$
\begin{aligned}
& \text { Let } \alpha:=\prod_{i=1}^{n} \alpha_{i}, \text { and for } \varepsilon>0 \text { let } \\
& \qquad D_{\varepsilon}:=\operatorname{diag}\left(1, \alpha_{1}-\varepsilon, \ldots, \prod_{i=1}^{n-1}\left(\alpha_{i}-\varepsilon\right)\right) .
\end{aligned}
$$

If

$$
k-1<\alpha k^{2}
$$

then (8) is contractive on $\mathbb{R}_{+}^{n}$ w.r.t. the scaled norm $\mid$. $\mid{ }_{1, D_{\varepsilon}}$ for all $\varepsilon>0$ sufficiently small. If $k-1=\alpha k^{2}$ then (8) does not satisfy (2), w.r.t. any norm, on $\mathbb{R}_{+}^{n}$, yet it is SOST on $\mathbb{R}_{+}^{n}$ w.r.t. the norm $|\cdot|_{1, D_{0}} \cdot{ }^{1}$

Note that for all $x \in \mathbb{R}_{+}^{n}$,

$$
g^{\prime}\left(x_{n}\right)=\frac{k-1}{\left(k+x_{n}\right)^{2}} \leq \frac{k-1}{k^{2}}=g^{\prime}(0) .
$$

Thus (9) implies that the system satisfies (2) if and only if the "total dissipation" $\alpha$ is strictly larger than $g^{\prime}(0)$.

Using the fact that $g(u)<1$ for all $u \geq 0$ it is straightforward to show that the set $\Omega_{r}:=r\left(\left[0, \alpha_{1}^{-1}\right] \times\right.$ $\left.\left[0,\left(\alpha_{1} \alpha_{2}\right)^{-1}\right] \times \cdots \times\left[0, \alpha^{-1}\right]\right)$ is an invariant set of the dynamics for all $r \geq 1$. Thus, (8), with $k-1 \leq$ $\alpha k^{2}$, admits a unique equilibrium point $e \in \Omega_{1}$ and $\lim _{t \rightarrow \infty} x(t, a)=e$, for all $a \in \mathbb{R}_{+}^{n}$. This property also follows from a more general result (Smith, 1995, Prop. 4.2.1) that is proved using the theory of irreducible cooperative dynamical systems. Yet the GCS approach leads to new insights. For example, it implies that the distance between trajectories can only decrease, and can also be used to prove entrainment to suitable generalizations of (8) that include periodically-varying inputs.

Cells often respond to external stimulus by modification of proteins. One mechanism for this is phosphorelay (also called phosphotransfer) in which a phosphate group is transferred through a serial chain of proteins from an initial histidine kinase (HK) down to a final response regulator (RR). The next example uses Theorem 1 to analyze a model for phosphorelay from (Csikasz-Nagy et al. (2011)).

Example 3 Consider the system

$$
\begin{aligned}
\dot{x}_{1} & =\left(p_{1}-x_{1}\right) c-\eta_{1} x_{1}\left(p_{2}-x_{2}\right), \\
\dot{x}_{2} & =\eta_{1} x_{1}\left(p_{2}-x_{2}\right)-\eta_{2} x_{2}\left(p_{3}-x_{3}\right), \\
& \vdots \\
\dot{x}_{n-1} & =\eta_{n-2} x_{n-2}\left(p_{n-1}-x_{n-1}\right)-\eta_{n-1} x_{n-1}\left(p_{n}-x_{n}\right), \\
\dot{x}_{n} & =\eta_{n-1} x_{n-1}\left(p_{n}-x_{n}\right)-\eta_{n} x_{n},
\end{aligned}
$$

1 Due to space limitations, the details of the analysis are placed at: http://arxiv.org/abs/1506.06613 
where $\eta_{i}, p_{i}>0$, and $c:\left[t_{1}, \infty\right) \rightarrow \mathbb{R}_{+}$. In the context of phosphorelay, $c(t)$ is the strength at time $t$ of the stimulus activating the $H K, x_{i}(t)$ is the concentration of the phosphorylated form of the protein at the ith layer at time $t$, and $p_{i}$ denotes the total protein concentration at that layer. Note that $\eta_{n} x_{n}$ is the flow of the phosphate group to an external receptor molecule.

In the particular case where $p_{i}=1$ for all $i$ (10) becomes the ribosome flow model (RFM) (Reuveni et al. (2011)). This is the mean-field approximation of an important model from non-equilibrium statistical physics called the totally asymmetric simple exclusion process (TASEP) (Blythe and Evans (2007)). In the RFM, $x_{i} \in[0,1]$ is the normalized occupancy at site $i$, where $x_{i}=0\left[x_{i}=1\right]$ means that site $i$ is completely free [full], and $\eta_{i}$ is the capacity of the link that connects site $i$ to site $i+1$. This has been used to model mRNA translation, where every site corresponds to a group of codons on the $m R N A$ strand, $x_{i}(t)$ is the normalized occupancy of ribosomes at site $i$ at time $t, c(t)$ is the initiation rate at time $t$, and $\eta_{i}$ $i$ s the elongation rate from site $i$ to site $i+1$.

Our original motivation for generalizing (2) was to prove entrainment in the RFM (Margaliot et al. (2014)). For more results on the RFM, see (Margaliot and Tuller (2012b, 2013, 2012a); Zarai et al. (2013); Poker et al. (2014)).

Assume that there exists $\eta_{0}>0$ such that $c(t) \geq \eta_{0}$ for all $t \geq t_{1}$. Let $\Omega:=\left[0, p_{1}\right] \times \cdots \times\left[0, p_{n}\right]$ denote the statespace of (10). Eq. (10) does not satisfy (2), w.r.t. any norm, on $\Omega$, yet it is SOST on $\Omega$ w.r.t. the $L_{1}$ norm. ${ }^{2}$

Considering Theorem 1 in the special case where all the sets $\Omega_{\zeta}$ in Definition 2 are equal to $\Omega$ yields the following result.

Corollary 1 Suppose that (1) is contractive on $\Omega$ w.r.t. a set of norms $|\cdot|_{\zeta}, \zeta \in(0,1 / 2]$, and that condition (c) in Definition 2 holds. Then (1) is SOST on $\Omega$ w.r.t. $|\cdot|$.

Corollary 1 may be useful in cases where some matrix measure of the Jacobian $J$ of (1) turns out to be non positive on $\Omega$, but not strictly negative, suggesting that the system is "on the verge" of satisfying (2). The next result demonstrates this for the time-invariant system

$$
\dot{x}=f(x),
$$

and the particular case of the matrix measure $\mu_{1}$ : $\mathbb{R}^{n \times n} \rightarrow \mathbb{R}$ induced by the $L_{1}$ norm. Recall that this is given by (3) with the $c_{j}$ s defined in (4).

\footnotetext{
2 Due to space limitations, the details of the analysis are placed at: http://arxiv.org/abs/1506.06613
}

Proposition 2 Consider the Jacobian $J(\cdot): \Omega \rightarrow \mathbb{R}^{n \times n}$ of the time-invariant system (11). Suppose that $\Omega$ is compact and that the set $\{1, \ldots, n\}$ can be divided into two non-empty disjoint sets $S_{0}$ and $S_{-}$such that the following properties hold for all $x \in \Omega$ :

(1) for any $k \in S_{0}, c_{k}(J(x)) \leq 0$;

(2) for any $j \in S_{-}, c_{j}(J(x))<0$;

(3) for any $i \in S_{0}$ there exists an index $z=z(i) \in S_{-}$ such that $J_{z i}(x)>0$.

Then (11) is SOST on $\Omega$ w.r.t. the $L_{1}$ norm.

The proof of Proposition 2 is based on the following idea. By compactness of $\Omega$, there exists $\delta>0$ such that

$$
c_{j}(J(x))<-\delta, \quad \text { for all } j \in S_{-} \text {and all } x \in \Omega \text {. }
$$

The conditions stated in the proposition imply that there exists a diagonal matrix $P$ such that $c_{k}\left(P J P^{-1}\right)<0$ for all $k \in S_{0}$. Furthermore, there exists such a $P$ with diagonal entries arbitrarily close to 1 , so $c_{j}\left(P J P^{-1}\right)<$ $-\delta / 2$ for all $j \in S_{-}$. Thus, $\mu_{1}\left(P J(x) P^{-1}\right)<0$ for all $x \in$ $\Omega$. Now Corollary 1 implies SOST. Note that this implies that the compactness assumption may be dropped if for example it is known that (12) holds.

Example 4 Consider the system:

$$
\begin{aligned}
& \dot{x}=-\delta x+k_{1} y-k_{2}\left(e_{T}-y\right) x, \\
& \dot{y}=-k_{1} y+k_{2}\left(e_{T}-y\right) x
\end{aligned}
$$

where $\delta, k_{1}, k_{2}, e_{T}>0$, and $\Omega:=[0, \infty) \times\left[0, e_{T}\right]$. This is a basic model for a transcriptional module that is ubiquitous in both biology and synthetic biology (see, e.g., Del Vecchio et al. (2008); Russo et al. (2010)). Here $x(t)$ is the concentration at time $t$ of a transcriptional factor $X$ that regulates a downstream transcriptional module by binding to a promoter with concentration $e(t)$ yielding a proteinpromoter complex $Y$ with concentration $y(t)$. The binding reaction is reversible with binding and dissociation rates $k_{2}$ and $k_{1}$, respectively. The linear degradation rate of $X$ is $\delta$, and as the promoter is not subject to decay, its total concentration, $e_{T}$, is conserved, so $e(t)=e_{T}-y(t)$. Russo et al. (2010) have shown that (13) is contractive w.r.t. a certain weighted $L_{1}$ norm. The Jacobian of (13) is $J=\left[\begin{array}{cc}-\delta-k_{2}\left(e_{T}-y\right) & k_{1}+k_{2} x \\ k_{2}\left(e_{T}-y\right) & -k_{1}-k_{2} x\end{array}\right]$, and all the properties in Prop. 2 hold with $S_{-}=\{1\}$ and $S_{0}=\{2\}$. Indeed, $J_{12}=k_{1}+k_{2} x>k_{1}>0$ for all $[x y]^{T} \in \Omega$. Thus, (13) is SOST on $\Omega$ w.r.t. the $L_{1}$ norm.

Arguing as in the proof of Proposition 2 for the matrix measure $\mu_{\infty}$ induced by the $L_{\infty}$ norm (see (5)) yields the following result. 
Proposition 3 Consider the Jacobian $J(\cdot): \Omega \rightarrow \mathbb{R}^{n \times n}$ of the time-invariant system (11). Suppose that $\Omega$ is compact and that the set $\{1, \ldots, n\}$ can be divided into two non-empty disjoint sets $S_{0}$ and $S_{-}$such that the following properties hold for all $x \in \Omega$ :

(1) $d_{j}(J(x)) \leq 0$ for all $j \in S_{0}$;

(2) $d_{k}(J(x))<0$ for all $k \in S_{-}$;

(3) for any $j \in S_{0}$ there exists an index $z=z(j) \in S_{-}$ such that $J_{j z}(x) \neq 0$.

Then (11) is SOST on $\Omega$ w.r.t. the $L_{\infty}$ norm.

\section{Contraction after a short overshoot}

A natural question is under what conditions SO and SOST are equivalent. To address this issue, we introduce the following definition.

Definition 3 We say that (1) is weakly expansive (WE) if for each $\delta>0$ there exists $\tau_{0}>0$ such that for all $a, b \in \Omega$ and all $t_{0} \geq 0$,

$$
\left|x\left(t, t_{0}, a\right)-x\left(t, t_{0}, b\right)\right| \leq(1+\delta)|a-b|,
$$

for all $t \in\left[t_{0}, t_{0}+\tau_{0}\right]$.

Proposition 4 Suppose that (1) is WE. Then (1) is SOST if and only if it is $S O$.

Remark 1 Suppose that $f$ in (1) is Lipschitz globally in $\Omega$ uniformly in $t$, i.e. there exists $L>0$ such that $|f(t, x)-f(t, y)| \leq L|x-y|$, for all $x, y \in \Omega, t \geq 0$. Then by Gronwall's Lemma (see, e.g. (Sontag, 1998, Appendix $C)),\left|x\left(t, t_{0}, a\right)-x\left(t, t_{0}, b\right)\right| \leq \exp \left(L\left(t-t_{0}\right)\right) \mid a-$ $b \mid$, for all $t \geq t_{0} \geq 0$, and this implies that (14) holds for $\tau_{0}:=\frac{1}{L} \ln (1+\delta)>0$. In particular, if $\Omega$ is compact and $f$ is periodic in $t$ then WE holds under rather weak continuity arguments on $f$.

\section{Contraction after a short transient}

For time-invariant systems whose state evolves on a convex and compact set it is possible to give a simple sufficient condition for ST. Let $\operatorname{Int}(S)[\partial S$ ] denote the interior [boundary] of a set $S$. We require the following definitions.

Definition 4 We say that (1) is non expansive (NE) w.r.t. a norm $|\cdot|$ if for all $a, b \in \Omega$ and all $s_{2}>s_{1} \geq 0$

$$
\left|x\left(s_{2}, s_{1}, a\right)-x\left(s_{2}, s_{1}, b\right)\right| \leq|a-b| .
$$

We say that (1) is weakly contractive (WC) if (15) holds with $\leq$ replaced by $<$.

Definition 5 The time-invariant system (11) with the state $x$ evolving on a compact and convex set $\Omega \subset \mathbb{R}^{n}$, is said to be interior contractive $(I C)$ w.r.t. a norm $|\cdot|$ : $\mathbb{R}^{n} \rightarrow \mathbb{R}_{+}$if the following properties hold: (a) for every $x_{0} \in \partial \Omega, x\left(t, x_{0}\right) \notin \partial \Omega$, for all $t>0$;

(b) for every $x \in \operatorname{Int}(\Omega)$,

$$
\mu(J(x))<0
$$

where $\mu: \mathbb{R}^{n \times n} \rightarrow \mathbb{R}$ is the matrix measure induced by $|\cdot|$.

In other words, the matrix measure is negative in the interior of $\Omega$, and the boundary of $\Omega$ is "repelling". Note that these conditions do not necessarily imply that the system satisfies $(2)$ on $\Omega$, as it is possible that $\mu(J(x))=$ 0 for some $x \in \partial \Omega$. Yet, (16) does imply that (11) is NE on $\Omega$. We can now state the main result in this subsection.

Theorem 2 If the system (11) is IC w.r.t. a norm | | | then it is $S T$ w.r.t. $|\cdot|$.

The proof of this result is based on showing that IC implies that for each $\tau>0$ there exists $d=d(\tau)>0$ such that $\operatorname{dist}\left(x\left(t, x_{0}\right), \partial \Omega\right) \geq d$, for all $x_{0} \in \Omega$ and all $t \geq \tau$, and then using this to conclude that for any $t \geq \tau$ all the trajectories of the system are contained in a convex and compact set $D \subset \operatorname{Int}(\Omega)$. In this set the system is contractive with rate $c:=\max _{x \in D} \mu(J(x))<0$. The next example, that is a variation of a system studied by Russo et al. (2010), demonstrates this reasoning.

Example 5 Consider a transcriptional factor $X$ that regulates a downstream transcriptional module by irreversibly binding, at a rate $k_{2}>0$, to a promoter $E$ yielding a protein-promoter complex $Y$. The promoter is not subject to decay, so its total concentration, denoted by $e_{T}>0$, is conserved. Assume also that $X$ is obtained from an inactive form $X_{0}$, for example through a phosphorylation reaction that is catalyzed by a kinase with abundance $u(t)$ satisfying $u(t) \geq u_{0}>0$ for all $t \geq 0$. The sum of the concentrations of $X_{0}, X$, and $Y$ is constant, denoted by $z_{T}$, with $z_{T}>e_{T}$. Letting $x_{1}(t), x_{2}(t)$ denote the concentrations of $X, Y$ at time $t$ yields the model

$$
\begin{aligned}
& \dot{x}_{1}=\left(z_{T}-x_{1}-x_{2}\right) u-\delta x_{1}-k_{2}\left(e_{T}-x_{2}\right) x_{1}, \\
& \dot{x}_{2}=k_{2}\left(e_{T}-x_{2}\right) x_{1}
\end{aligned}
$$

with the state evolving on $\Omega:=\left[0, z_{T}\right] \times\left[0, e_{T}\right]$. Here $\delta \geq 0$ is the dephosphorylation rate $X \rightarrow X_{0}$. Let $P:=\left[\begin{array}{ll}1 & 1 \\ 0 & 1\end{array}\right]$, and consider the matrix measure $\mu_{\infty, P}$. Let $\tilde{J}:=$ PJP $P^{-1}$. A calculation yields $\tilde{J}=$ $\left[\begin{array}{cc}-u-\delta & \delta \\ k_{2}\left(e_{T}-x_{2}\right) & k_{2}\left(x_{2}-x_{1}-e_{T}\right)\end{array}\right]$, so $d_{1}(\tilde{J})=-u-\delta+$ 
$|\delta| \leq-u_{0}<0$, and

$$
\begin{aligned}
d_{2}(\tilde{J}) & =k_{2}\left(x_{2}-x_{1}-e_{T}\right)+\left|k_{2}\left(e_{T}-x_{2}\right)\right| \\
& =-k_{2} x_{1} .
\end{aligned}
$$

Letting $S:=\{0\} \times\left[0, e_{T}\right]$, we conclude that $\mu_{\infty, P}(x)<0$ for all $x \in(\Omega \backslash S)$. For any $x \in S, \dot{x}_{1}=\left(z_{T}-x_{2}\right) u \geq$ $\left(z_{T}-e_{T}\right) u_{0}>0$, and arguing as in the proof of Theorem 2, we conclude that for any $\tau>0$ there exists $d=$ $d(\tau)>0$ such that $x_{1}(t, a) \geq d$, for all $a \in \Omega$ and all $t \geq \tau$. In other words, after time $\tau$ all the trajectories are contained in the closed and convex set $D=D(\tau):=$ $\left[d, z_{T}\right] \times\left[0, e_{T}\right]$. Letting $c:=c(\tau)=\max _{x \in D} \mu_{\infty, P}(J(x))$ yields $c<0$ and $|x(t+\tau, a)-x(t+\tau, b)|_{\infty, P} \leq \exp (c t) \mid a-$ $\left.b\right|_{\infty, P}$ for all $a, b \in \Omega$ and all $t>0$, so (17) is $S T$ w.r.t. $|\cdot|_{\infty, P}$.

As noted above, the introduction of GCS is motivated by the idea that contraction is used to prove asymptotic results, so allowing initial transients should increase the class of systems that can be analyzed while still allowing to prove asymptotic results. The next result demonstrates this.

Corollary 2 If (11) is IC with respect to some norm then it admits a unique equilibrium point $e \in \operatorname{Int}(\Omega)$, and $\lim _{t \rightarrow \infty} x(t, a)=e$ for all $a \in \Omega$.

Remark 2 Consider the variational system (see, e.g., Forni and Sepulchre (2014)) associated with (11):

$$
\begin{aligned}
\dot{x} & =f(x), \\
\dot{\delta} x & =J(x) \delta x .
\end{aligned}
$$

Our proof of Corollary 2 is based on Theorem 2. An alternative proof is possible, using the Lyapunov-Finsler function $V(x, \delta x):=|\delta x|$, where $|\cdot|: \mathbb{R}^{n} \rightarrow \mathbb{R}_{+}$is the vector norm corresponding to the matrix measure $\mu$ in (16), and the LaSalle invariance principle described in (Forni and Sepulchre (2014)).

Contraction can be characterized using a LyapunovFinsler function (Forni and Sepulchre (2014)). The next result describes a similar characterization for ST. For simplicity, we state this for the time-invariant system (11).

Proposition 5 The following two conditions are equivalent.

(a) The time-invariant system (11) is ST w.r.t. a norm|. I.

(b) For any $\tau>0$ there exists $\ell=\ell(\tau)>0$ such that for any $a, b \in \Omega$ and any $c$ on the line connecting $a$ and $b$ the solution of $(18)$ with $x(0)=c$ and $\delta x(0)=b-a$ satisfies $|\delta x(t+\tau)| \leq \exp (-\ell t)|\delta x(0)|$, for all $t \geq 0$.
Note that the latter condition implies that the function $V(x, \delta x):=|\delta x|$ is a generalized Lyapunov-Finsler function in the following sense. For any $\tau>0$ there exists $\ell=\ell(\tau)>0$ such that along solutions of the variational system: $V(x(t+\tau, x(0)), \delta x(t+\tau, \delta x(0), x(0))) \leq$ $\exp (-\ell t) V(x(0), \delta x(0))$, for all $t \geq 0$.

It is straightforward to show that each of the three generalizations of contraction in Definition 1 implies that (1) is NE. One may perhaps expect that any of the three generalizations of contraction in Definition 1 also implies WC. Indeed, ST does imply WC, because $\left|x\left(s_{2}, s_{1}, a\right)-x\left(s_{2}, s_{1}, b\right)\right| \leq \exp \left(-\ell\left(s_{2}-s_{1}\right) / 2\right)|a-b|<$ $|a-b|$, for all $0 \leq s_{1}<s_{2}$ if ST holds (simply apply the definition with $t_{1}=s_{1}, \tau=\left(s_{2}-s_{1}\right) / 2>0$, and $t_{2}=s_{1}+\tau$ in (6)). However, the next example shows that SO does not imply WC.

Example 6 Consider the scalar system

$$
\dot{x}= \begin{cases}-2 x, & 0 \leq|x|<1 / 2 \\ -\frac{x}{|x|}, & \frac{1}{2} \leq|x| \leq 1\end{cases}
$$

with $x$ evolving on $\Omega:=[-1,1]$. Clearly, this system is not WC. However, it is not difficult to show that it satisfies the definition of $S O$ with $\ell=\ell(\varepsilon):=\min \{\ln (1+$ $\varepsilon), 1\}$.

Fig. 1 summarizes the relations between the various contraction notions.

\section{Acknowledgments}

We thank Zvi Artstein and George Weiss for helpful comments. We are grateful to the anonymous reviewers for many helpful comments.

\section{References}

Aminzare, Z. and Sontag, E. D. (2013). Logarithmic Lipschitz norms and diffusion-induced instability. Nonlinear Analysis: Theory, Methods \& Applications, 83, 31-49.

Aminzare, Z. and Sontag, E. D. (2014). Contraction methods for nonlinear systems: A brief introduction and some open problems. In Proc. 53rd IEEE Conf. on Decision and Control, pages 3835-3847, Los Angeles, CA.

Angeli, D. (2002). A Lyapunov approach to incremental stability properties. IEEE Trans. Automat. Control, 47, 410-421.

Arcak, M. (2011). Certifying spatially uniform behavior in reaction-diffusion $\mathrm{PDE}$ and compartmental ODE systems. Automatica, 47(6), 1219-1229.

Aylward, E. M., Parrilo, P. A., and Slotine, J.-J. E. (2008). Stability and robustness analysis of nonlinear 


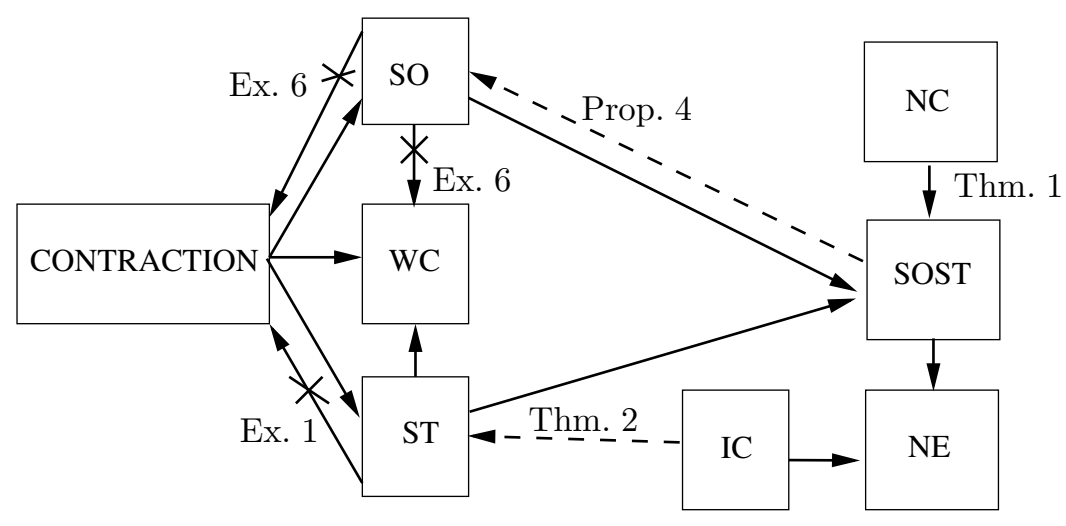

Fig. 1. Relations between various contraction notions. An arrow denotes implication; a crossed out arrow denotes that the implication is in general false; and a dashed arrow denotes an implication that holds under an additional condition. Some of the relations are immediate. Others follow from the results marked near the arrows.

systems via contraction metrics and SOS programming. Automatica, 44(8), 2163-2170.

Blythe, R. A. and Evans, M. R. (2007). Nonequilibrium steady states of matrix-product form: a solver's guide. J. Phys. A: Math. Theor., 40(46), R333-R441.

Bonnabel, S., Astolfi, A., and Sepulchre, R. (2011). Contraction and observer design on cones. In Proc. 50th IEEE Conf. on Decision and Control and European Control Conference, pages 7147-7151, Orlando, Florida.

Csikasz-Nagy, A., Cardelli, L., and Soyer, O. S. (2011). Response dynamics of phosphorelays suggest their potential utility in cell signaling. J. Royal Society Interface, 8, 480-488.

Del Vecchio, D., Ninfa, A. J., and Sontag, E. D. (2008). Modular cell biology: Retroactivity and insulation. Molecular Systems Biology, 4(1), 161.

Desoer, C. and Haneda, H. (1972). The measure of a matrix as a tool to analyze computer algorithms for circuit analysis. IEEE Trans. Circuit Theory, 19, 480486.

Dorfler, F. and Bullo, F. (2012). Synchronization and transient stability in power networks and nonuniform Kuramoto oscillators. SIAM J. Control Optim., 50, 1616-1642.

Forni, F. and Sepulchre, R. (2014). A differential Lyapunov framework for contraction analysis. IEEE Trans. Automat. Control, 59(3), 614-628.

Jouffroy, J. (2005). Some ancestors of contraction analysis. In Proc. 44th IEEE Conf. on Decision and Control, pages 5450-5455, Seville, Spain.

Lewis, D. C. (1949). Metric properties of differential equations. American J. Math., 71(2), 294-312.

Lohmiller, W. and Slotine, J.-J. E. (1998). On contraction analysis for non-linear systems. Automatica, 34, 683-696.

Lohmiller, W. and Slotine, J.-J. E. (2000). Control system design for mechanical systems using contraction theory. IEEE Trans. Automat. Control, 45, 984-989.

Margaliot, M. and Tuller, T. (2012a). On the steadystate distribution in the homogeneous ribosome flow model. IEEE/ACM Trans. Computational Biology and Bioinformatics, 9, 1724-1736.

Margaliot, M. and Tuller, T. (2012b). Stability analysis of the ribosome flow model. IEEE/ACM Trans. Computational Biology and Bioinformatics, 9, 1545-1552.

Margaliot, M. and Tuller, T. (2013). Ribosome flow model with positive feedback. J. Royal Society Interface, 10, 20130267.

Margaliot, M., Sontag, E. D., and Tuller, T. (2014). Entrainment to periodic initiation and transition rates in a computational model for gene translation. PLoS ONE, 9(5), e96039.

Pham, Q.-C., Tabareau, N., and Slotine, J.-J. (2009). A contraction theory approach to stochastic incremental stability. IEEE Trans. Automat. Control, 54, 816820 .

Poker, G., Zarai, Y., Margaliot, M., and Tuller, T. (2014). Maximizing protein translation rate in the nonhomogeneous ribosome flow model: A convex optimization approach. J. Royal Society Interface, 11(100), 20140713.

Reuveni, S., Meilijson, I., Kupiec, M., Ruppin, E., and Tuller, T. (2011). Genome-scale analysis of translation elongation with a ribosome flow model. PLoS Computational Biology, 7, e1002127.

Rüffer, B. S., van de Wouw, N., and Mueller, M. (2013). Convergent systems vs. incremental stability. Systems Control Lett., 62, 277-285.

Russo, G., di Bernardo, M., and Sontag, E. D. (2010). Global entrainment of transcriptional systems to periodic inputs. PLoS Computational Biology, 6, e1000739.

Russo, G., di Bernardo, M., and Sontag, E. D. (2013). A contraction approach to the hierarchical analysis and design of networked systems. IEEE Trans. Automat. Control, 58, 1328-1331.

Simpson-Porco, J. W. and Bullo, F. (2014). Contraction theory on Riemannian manifolds. Systems Control Lett., 65, 74-80.

Slotine, J.-J. E. (2003). Modular stability tools for distributed computation and control. Int. J. Adaptive 
Control and Signal Processing, 17, 397-416.

Smith, H. L. (1995). Monotone Dynamical Systems: An Introduction to the Theory of Competitive and Cooperative Systems, volume 41 of Mathematical Surveys and Monographs. Amer. Math. Soc., Providence, RI.

Soderlind, G. (2006). The logarithmic norm. History and modern theory. BIT Numerical Mathematics, 46, 631-652.

Sontag, E. D. (1998). Mathematical Control Theory: Deterministic Finite-Dimensional Systems, volume 6 of Texts in Applied Mathematics. Springer-Verlag, New York, 2 edition.

Sontag, E. D., Margaliot, M., and Tuller, T. (2014). On three generalizations of contraction. In Proc. 53rd IEEE Conf. on Decision and Control, pages 15391544, Los Angeles, CA.

Vidyasagar, M. (1978). Nonlinear Systems Analysis. Prentice Hall, Englewood Cliffs, NJ.

Wang, W. and Slotine, J. J. (2005). On partial contraction analysis for coupled nonlinear oscillators. Biol. Cybern., 92, 38-53.

Zamani, M., van de Wouw, N., and Majumdar, R. (2013). Backstepping controller synthesis and characterizations of incremental stability. Systems Control Lett., 62(10), 949-962.

Zarai, Y., Margaliot, M., and Tuller, T. (2013). Explicit expression for the steady state translation rate in the infinite-dimensional homogeneous ribosome flow model. IEEE/ACM Trans. Computational Biology and Bioinformatics, 10, 1322-1328. 